\title{
Retrospective Study of Real-World Treatment Patterns and Outcomes in Advanced/Metastatic Renal Cell Carcinoma Patients Receiving Lenvatinib/ Everolimus after Heavy Pretreatment ${ }^{1}$
}

Nicholas J. Vogelzang ${ }^{\mathrm{a}}$, Alisha M. Monnette ${ }^{\mathrm{b}, *}$, Yunfei Wang ${ }^{\mathrm{b}}$, Yin Wan ${ }^{\mathrm{c}}$, Nicholas J. Robert ${ }^{\mathrm{b}}$ and Nizar M. Tannir ${ }^{\mathrm{d}}$

${ }^{a}$ US Oncology Comprehensive Cancer Centers of Nevada, Las Vegas, NV, USA

${ }^{\mathrm{b}}$ Ontada, Woodlands, TX, USA

${ }^{\mathrm{c}}$ Eisai Inc. Woodcliff Lake, NJ, USA

${ }^{\mathrm{d}}$ MD Anderson Cancer Center, Houston, TX, USA

Received 7 July 2021

Accepted 27 August 2021

Pre-press 18 September 2021

Published 3 December 2021

\begin{abstract}
.
BACKGROUND: Lenvatinib with everolimus ("Len/Eve") is approved for advanced/metastatic RCC following one antiangiogenic therapy.

OBJECTIVE: This study evaluated patient characteristics, treatment patterns and overall survival (OS) with second-line or later $(2 \mathrm{~L}+) \mathrm{Len} /$ Eve for advanced/metastatic RCC.

METHODS: A retrospective observational study was conducted using electronic health records. Adult patients who initiated 2L+ Len/Eve for advanced/metastatic RCC from May 13, 2016 to July 31, 2019 were included. Patient characteristics and treatment patterns were assessed across the overall population and by post-immuno-oncology (IO) and post-tyrosine kinase inhibitors (TKI) groups. OS was estimated from Len/Eve initiation (i.e., index date) using Kaplan-Meier.

RESULTS: Among the study population $(n=152), 44.1 \%$ received $2 \mathrm{~L} / 3 \mathrm{~L} \mathrm{Len/Eve} \mathrm{and} \mathrm{median} \mathrm{number} \mathrm{of} \mathrm{prior} \mathrm{therapies}$ was 3 (range:1-8). Median age was 63.2 years, $78.9 \%$ were Caucasian, $73.7 \%$ were male, and 56.6\% had ECOG performance status 0/1. At initial diagnosis, 65.8\% had stage IV disease. At index, 53.3\% had an International Metastatic RCC Database Consortium risk score of intermediate/poor, $15.1 \%$ favorable, and 31.6\% missing score. Sixty-five (42.8\%) received IO-based regimens and $49.3 \%$ received TKIs directly before index. Median OS from index was 13.9 (95\% CI: 9.5-16.5) months and 2L/3L and 4L+ were 12.1 (95\% CI: 8.4-17.0) and 14.8 (95\% CI: 8.9-22.5) months, respectively. Median OS for Len/Eve post-IO and post-TKI were 13.9 (95\% CI: 8.4-21.3) and 14.8 (95\% CI: 7.8-16.5) months, respectively.
\end{abstract}

\footnotetext{
${ }^{1}$ Previous presentations: This work has been presented as a poster presentation at the IKCS 2020 conference, November 6th, 2020 held virtually.

${ }^{*}$ Correspondence to: Alisha Monnette, 10101 Woodloch Forest Dr., The Woodlands, TX 77380, USA. Tel.: +1 770462 7590; E-mail: alisha.monnette@mckesson.com.
} 
Conclusion: This study suggested that $2 \mathrm{~L}+\mathrm{Len} / \mathrm{Eve}$ has clinical effectiveness for advanced/metastatic RCC in a US community oncology setting. Future studies are needed to confirm the association of improved survival with $2 \mathrm{~L}+\mathrm{Len} /$ Eve.

Keywords: Immuno-oncology, tyrosine kinase inhibitors, overall survival, non-clear cell, clinical effectiveness

\section{INTRODUCTION}

Renal cell carcinoma (RCC) is the sixth most common cancer in men and the ninth most common cancer in women, accounting for $3 \%$ to $5 \%$ of all adult malignancies [1]. An estimated 76,080 new cases of RCC and 13,780 deaths from RCC will have occurred in the United States (US) in 2021. At the initial diagnosis, approximately $65 \%$ of patients have localized disease, $16 \%$ have regional spread, and $16 \%$ have distant metastasis. The 5-year survival for patients with RCC is $75.2 \%$; however, patients with advanced or metastatic RCC (advanced/metastatic RCC) have a poorer prognosis (14\%) compared to localized RCC (93\%) (during 2011-2017) [2].

The treatment landscape for advanced/metastatic RCC continues to evolve. Immuno-oncology (IO)based therapies and tyrosine kinase inhibitors (TKI)-based therapies including lenvatinib, sunitinib, pazopanib and cabozantinib are FDA approved for first-line treatments and axitinib, cabozantinib, nivolumab, lenvatinib plus everolimus, and everolimus monotherapy among others for second (2L)- and subsequent-line treatments [3-5]. The combination of lenvatinib plus everolimus (Len/Eve) was approved by the Food and Drug Administration in May of 2016 for advanced/metastatic RCC following one prior antiangiogenic therapy [6]. This approval was based on results from a randomized, phase 2, open-label, multi-center trial (Study 205). In this trial, 51 patients received Len/Eve and 50 patients received single agent everolimus. Len/Eve combination therapy was associated with significantly longer progression-free survival compared with everolimus alone (median 14.6 months [95\% CI 5.9-20.1] vs 5.5 months [3.5-7.1]; hazard ratio [HR] 0.40, 95\% CI $0.24-0.68 ; p=0.0005$ ) [7].

While several studies have explored the clinical outcomes of patients receiving cabozantinib or nivolumab monotherapy as second-line or subsequent lines of treatment $(2 \mathrm{~L}+)$ in a real-world setting [8-12], data on clinical outcomes are limited for patients with advanced/metastatic RCC treated with $2 \mathrm{~L}+\mathrm{Len} /$ Eve post antiangiogenic or IO-based therapy. In 2020, a case report by Hamieh et al. (2020) found that seven advanced/metastatic RCC patients with primary resistance to first-line therapies benefitted from subsequent Len/Eve combination therapy [13]. Due to the small sample size in this case report, it is evident that additional studies evaluating Len/Eve use are necessary to assist clinicians and patients in choosing the optimal treatment. Therefore, this study aims to understand the patient characteristics, treatment patterns and clinical outcomes of patients with advanced/metastatic RCC who received 2L combination therapy of Len/Eve in US community oncology practices.

\section{PATIENTS AND METHODS}

\section{Study design and patient population}

This was a retrospective observational cohort study that examined demographics, clinical characteristics, treatment patterns and outcomes for patients with advanced/metastatic RCC treated with $2 \mathrm{~L}+\mathrm{Len} / \mathrm{Eve}$ between May 13, 2016 and July 31, 2019. May 13, 2016 was chosen as the lower bound of the index identification period because it was the date that Len/Eve combination therapy received FDA approval for advanced/metastatic RCC. July 31, 2019 was chosen as the upper bound to allow at least 6 months of follow up prior to the anticipated data cut rate on January 31,2020 . Patients were followed until January 31,2020 or last patient record (Fig. 1).

Eligible patients were 18 years of age or older at the index date and had a documented diagnosis of advanced/metastatic RCC, initiated Len/Eve between May 13, 2016 and July 31, 2019, received care at US Oncology Network or Onmark clinics utilizing the full electronic healthcare record (EHR) capacities at the time of treatment, and had $\geq 2$ office visits during the study observation period. Patients were excluded if they were enrolled in clinical trials investigating Len/Eve or being treated for other types of primary tumors during the index period.

The date of initiation of Len/Eve was defined as the index date. The pre-index period was the time period from the initial date of advanced/metastatic RCC diagnosis until (but not including) the index date. The post-index period was the time period from the index date until the final clinical visit, death, or 


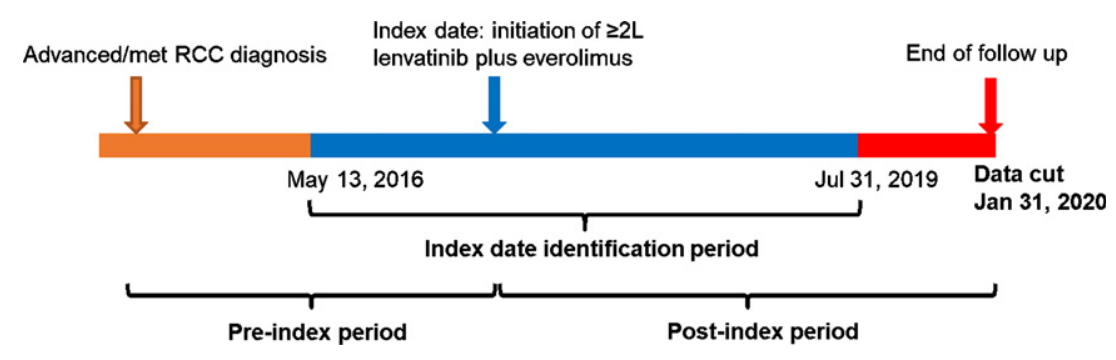

Fig. 1. Overview of study design.

end of study date, whichever came first. During the post-index period, patients had variable follow-up durations as they were each followed longitudinally until last patient record or end of the study period.

\section{Data source}

Data were captured from The US Oncology Network and Onmark from records dating from May 13, 2016 to January 31, 2020. The US Oncology Network includes more than 1,400 physicians operating in more than 470 sites of care across 25 states and provides care for nearly 1 million patients with cancer each year. The EHR of the US Oncology Network, iKnowMed (iKM), was used in this study. In addition, data were included from approximately 80 Onmark clinics that have adopted the iKM EHR. iKM is an oncology-specific EHR system that captures outpatient practice encounter histories for patients under community-based care, including but not limited to patient demographics such as age and gender; clinical information such as disease diagnosis, diagnosis stages, performance status information and laboratory testing results; and treatment information, such as treatment administration within The US Oncology Network and Onmark clinics.

To supplement available vital status information in iKM, the Social Security Death Index (SSDI) was used to estimate OS. OS was defined as the interval from the index date to the date of death as documented in the SSDI and iKM EHR database. Patients alive at the end of the data collection period were censored on the study end date or the last visit date available in the database, whichever occurred first. Because the study derived data from the iKM database to meet the objectives, an intent-to-treat perspective was applied. All study data were handled according to the Health Insurance Portability and Accountability Act and the Health Information Technology for Economic and Clinical Health Act. The study protocol received an exception and waiver of informed consent from the US Oncology Institutional Review Board, IRB\# 20-004E.

\section{Patient characteristics and treatment patterns}

Patient demographics examined in this study included age, gender, race, ethnicity, geographic region, and smoking status. Available data were obtained on clinical characteristics, International Metastatic RCC Database Consortium (IMDC) risk status, and treatment details. Treatment sequences were determined based on the start and stop dates of regimens. The treatment episode was defined within 28 days. If a gap existed that was longer than a specific time window (i.e., $\geq 90$ days), the line of therapy (LOT) was defined as the next LOT even with the same treatment. Cancelled drug orders and administrations were not used to determine the LOT.

\section{Statistical analysis}

Demographics and clinical characteristics, treatment patterns, and outcomes were determined for all study patients and for the subgroups treated with Len/Eve post-IO (i.e., including IO monotherapy and IO-based regimen) or post-TKI. Classification of patients into those subgroups was based on the treatment regimen(s) immediately before Len/Eve. For example, if a patient began nivolumab monotherapy and advanced to a new LOT with Len/Eve, that patient was assigned to the post-IO subgroup. All patients were followed from index date until end of study period.

Demographic and clinical characteristics at baseline and treatment patterns were analyzed descriptively for the overall study population. Categorical variables (e.g., Eastern Cooperative Oncology group [ECOG] performance status) were reported as frequencies and percentages. Continuous variables such as age were reported as mean, standard deviation, median, and range (minimum-maximum). In the case 


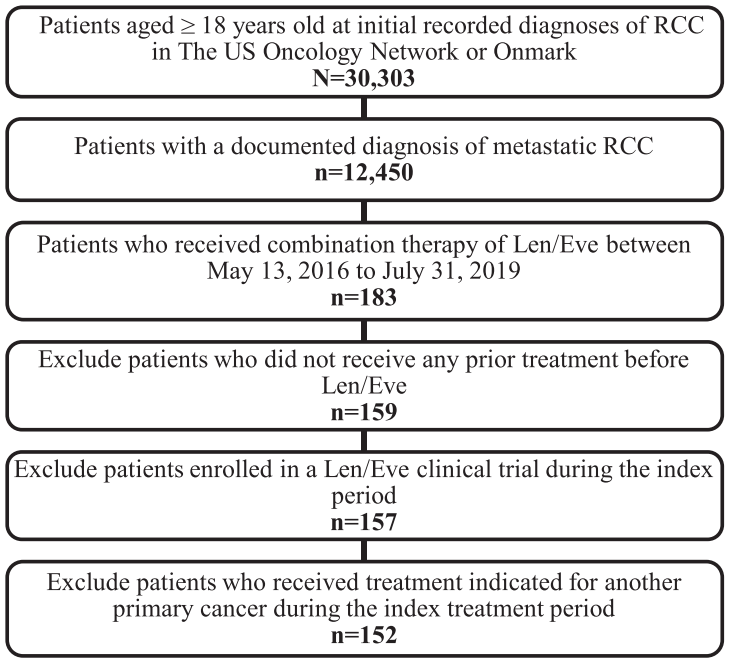

Fig. 2. Study attrition. Abbreviations: LOT, line of therapy; RCC, renal cell carcinoma.

of missing observations, the number and percentage of missing values was reported. Overall survival (OS) was the primary clinical endpoint. OS was defined as the interval between the initiation of the index treatment to the date of death and measured using the Kaplan-Meier method with $95 \%$ confidence intervals (CIs). Patients still alive by the end of the study observation period were censored on the study end date or the last visit date reported in the dataset, whichever happened first. OS probabilities were reported for $6,12,18$, and 24 months. Results were reported in aggregate using SAS $^{\circledR} 9.4$ (SAS Institute Inc., Cary, NC, US).

\section{RESULTS}

\section{Patient demographic and clinical characteristics}

A total of 152 patients were included in the study population and had been diagnosed with advanced/metastatic RCC at a median of 22.9 (IQR $13.8,37.0)$ months before the index date. The median age was 63.4 years, $73.7 \%$ were male, $78.9 \%$ identified as Caucasian, $41.4 \%$ received care in the western U.S. region and $49.3 \%$ had never smoked (Table 1). At initial diagnosis, $65.8 \%$ of patients had stage IV disease and $9.2 \%$ had non-clear cell carcinoma. The lung was the most common site for distant metastases (35.5\%), followed by the bones $(18.4 \%)$, and lymph nodes $(13.2 \%)$. Approximately $56 \%$ of the patients had an ECOG performance status score of 0 or 1 . At the time of starting Len/Eve, patients were predom- inately IMDC intermediate risk with more than half $(52.0 \%)$ at intermediate risk, $15.1 \%$ at favorable risk, only 2 patients $(1.3 \%)$ at poor risk, and $31.6 \%$ with a missing score.

\section{Treatment patterns}

Sixty-seven patients (44.0\%) received Len/Eve in the second- or third-line setting, while 85 patients $(55.9 \%)$ received Len/Eve in the fourth-line or later setting. The median number of prior therapies was 3 (range: 1-8). Prior to starting treatment with Len/Eve, approximately $42.8 \%$ received an IO-based regimen (including IO-IO, IO-TKI, and IO monotherapies) and $49.3 \%$ received TKI treatments alone directly before index (Table 2).

\section{Clinical outcomes}

Among all patients, median OS from index date was 13.9 months (95\% CI: 9.5-16.5); the 6- and 12-month survival probabilities were $70.8 \%(95 \%$ CI: $62.5-77.6)$ and $52.7 \%$ (95\% CI: 43.4-61.1), respectively (Table 3 ). Patients receiving Len/Eve as 2L/3L had an OS of 12.1 months (CI: 8.4-17.0), and those receiving Len/Eve during lines $4 \mathrm{~L}+$ was 14.8 months (CI: 8.9-22.5) (Table 3). The median OS for patients in the post-IO and post-TKI subgroups were 13.9 months (95\% CI: 8.4-21.3) and 14.8 (95\% CI: 7.8-16.5) months, respectively (Table 3 ).

\section{DISCUSSION}

As advanced/metastatic RCC has a poorer prognosis than localized RCC, it is crucial that clinical outcomes for treatments post clinical trials are available to aid providers in decision making. Clinical decision making is based on clinical trials, post clinical trial data (e.g., personal experience), and real-world data such as those reported here. Realworld data give valuable information on clinical outcomes, treatment patterns, and safety in clinical practice, which includes patients with characteristics not necessarily included in clinical trials (e.g., sicker patients). Although Len/Eve was FDA approved for the second-line or later-line $(2 \mathrm{~L}+)$ treatment of advanced or metastatic RCC in 2016, there is limited real-world data or research evidence to inform clinicians of the outcomes of patients with RCC who receive Len/Eve in the $2 \mathrm{~L}+$ setting [14]. To the best of our knowledge, this is the largest retrospective study that examines patient characteristics, 
Table 1

Baseline demographic and clinical characteristics for patients with advanced/metastatic RCC treated with $2 \mathrm{~L}+\mathrm{Len} /$ Eve

\begin{tabular}{|c|c|}
\hline Baseline characteristics & $\mathrm{N}=152$ \\
\hline \multicolumn{2}{|l|}{ Age at index (years) } \\
\hline Median (range) & $63(33,91)$ \\
\hline \multicolumn{2}{|l|}{ Gender $-n(\%)$} \\
\hline Male & $112(73.7)$ \\
\hline Female & $40(26.3)$ \\
\hline \multicolumn{2}{|l|}{ Race $-n(\%)$} \\
\hline Caucasian & $120(78.9)$ \\
\hline African American & $6(3.9)$ \\
\hline Asian & $4(2.6)$ \\
\hline Other & $15(9.9)$ \\
\hline Not documented & $7(4.6)$ \\
\hline \multicolumn{2}{|l|}{ Geographic region $-n(\%)$} \\
\hline West & $63(41.4)$ \\
\hline South & $48(31.6)$ \\
\hline Midwest & $39(25.7)$ \\
\hline Northeast & $2(1.3)$ \\
\hline \multicolumn{2}{|l|}{ BMI category at index treatment initiation $-\mathrm{n}(\%)$} \\
\hline Underweight $\left(<18 \mathrm{~kg} / \mathrm{m}^{2}\right)$ & $2(1.3)$ \\
\hline Normal $\left(18-24.9 \mathrm{~kg} / \mathrm{m}^{2}\right)$ & $52(34.2)$ \\
\hline Overweight $\left(25-29.9 \mathrm{~kg} / \mathrm{m}^{2}\right)$ & $47(30.9)$ \\
\hline Obese $\left(30+\mathrm{kg} / \mathrm{m}^{2}\right)$ & $46(30.3)$ \\
\hline Not documented & $5(3.3)$ \\
\hline \multicolumn{2}{|l|}{ Smoking status $-n(\%)$} \\
\hline Never & $75(49.3)$ \\
\hline Former & $52(34.2)$ \\
\hline Current & $17(11.2)$ \\
\hline Not documented & $8(5.3)$ \\
\hline \multicolumn{2}{|c|}{ Time since initial RCC diagnosis to metastatic disease (months) } \\
\hline Patients with available data & 152 \\
\hline Mean (SD) & $25.0(56.5)$ \\
\hline Median (Min, Max) & $2.4(0.03,379.7)$ \\
\hline \multicolumn{2}{|c|}{ Time since advanced/metastatic RCC diagnosis to index date (months) } \\
\hline Patients with available data & 152 \\
\hline Mean (SD) & $29.0(22.7)$ \\
\hline Median (Min, Max) & $22.9(2.3,133.0)$ \\
\hline \multicolumn{2}{|l|}{ Stage at initial RCC diagnosis $-n(\%)$} \\
\hline I & $11(7.2)$ \\
\hline II & $11(7.2)$ \\
\hline III & $16(10.5)$ \\
\hline IV & $100(65.8)$ \\
\hline Not documented & $14(9.2)$ \\
\hline \multicolumn{2}{|l|}{ Histology at initial RCC diagnosis $-n(\%)$} \\
\hline Clear cell carcinoma & $124(81.6)$ \\
\hline Non-clear cell carcinoma & $14(9.2)$ \\
\hline Papillary carcinoma & $8(5.3)$ \\
\hline Chromophobe carcinoma & $2(1.3)$ \\
\hline Collecting duct & $1(0.7)$ \\
\hline Renal medullary carcinoma & $1(0.7)$ \\
\hline Translocation & $1(0.7)$ \\
\hline Unclassified & $1(0.7)$ \\
\hline Not Documented & $14(9.2)$ \\
\hline \multicolumn{2}{|l|}{ Sarcomatoid features $-n(\%)$} \\
\hline Yes & $7(4.6)$ \\
\hline No & $77(50.7)$ \\
\hline Not documented & $68(44.7)$ \\
\hline Distant metastatic site $(\mathrm{s}), n(\%)^{\mathrm{a}}$ & \\
\hline Lung & $54(35.5)$ \\
\hline Bone & $28(18.4)$ \\
\hline Lymph & $20(13.2)$ \\
\hline Liver & $9(5.9)$ \\
\hline Brain & $2(1.3)$ \\
\hline Other & $43(28.3)$ \\
\hline ECOG PS $-n(\%)^{\mathrm{b}}$ & \\
\hline 0 & $20(13.2)$ \\
\hline 1 & $86(56.6)$ \\
\hline 2 & $29(19.1)$ \\
\hline 3 & $2(1.3)$ \\
\hline Not documented & $15(9.9)$ \\
\hline IMDC score $-n(\%)^{\mathrm{b}}$ & \\
\hline Favorable & $23(15.1)$ \\
\hline Intermediate & $79(52.0)$ \\
\hline Poor & $2(1.3)$ \\
\hline Not documented & $48(31.6)$ \\
\hline
\end{tabular}

Abbreviations: advanced/metastatic RCC, advanced/metastatic renal cell carcinoma; ECOG PS, Eastern Cooperative Oncology Group performance status; IMDC, International Metastatic RCC Database Consortium; SD, standard deviation. ${ }^{\text {a At }} 60$ days before diagnosis of advanced/metastatic RCC. ${ }^{\mathrm{b}}$ At 60 days before initiation of Len + Eve. 
Table 2

Baseline treatment characteristics for patients with advanced/metastatic RCC treated with $2 \mathrm{~L}+\mathrm{Len}+$ Eve

\begin{tabular}{lc}
\hline \multicolumn{1}{c}{ Baseline characteristics } & $\mathrm{N}=152$ \\
\hline Follow-up duration from initiation of index treatment (months) & \\
Patients with available data & 152 \\
Mean (SD) & $10.2(8.5)$ \\
Median (Min, Max) & $7.5(0.2,43.8)$ \\
Number of prior treatments & \\
$\quad$ Mean (SD) & $3.0(1.6)$ \\
Median (Min, Max) & $3(1-8)$ \\
Line of therapy for index treatment (i.e., Len/Eve) - $n(\%)^{\mathrm{a}}$ & \\
2 & $21(13.8)$ \\
3 & $46(30.3)$ \\
4 & $41(27.0)$ \\
5 & $21(13.8)$ \\
6 & $8(5.3)$ \\
$\geq 7$ & $15(9.9)$ \\
Starting dose of index treatment: lenvatinib - $n(\%)$ & \\
18 & $112(73.7)$ \\
$<18$ & $40(26.3)$ \\
Starting dose of index treatment: everolimus $-n(\%)$ & \\
5 & $135(88.8)$ \\
10 & $14(9.2)$ \\
7.5 & $2(1.3)$ \\
2.5 & $1(0.7)$ \\
Lenvatinib + everolimus treatment discontinuation rate - $n(\%)$ & $122(80.3)$ \\
Treatment regimen immediately prior to index - n $(\%)$ & \\
IO-TKI & $24(15.8)$ \\
TKI only & $75(49.3)$ \\
TO monotherapy or IO-IO combo & $41(27.0)$ \\
\hline Abbreviations: advanced/metastatic RCC, advanced/metastatic renal cell carcinoma; IO, \\
immuno-oncology therapies; SD, standard deviation; TKI, tyrosine kinase inhibitors. \\
\end{tabular}

Table 3

Median OS in overall population and stratified by prior treatments and line of therapy

\begin{tabular}{|c|c|c|c|c|c|}
\hline \multirow[b]{2}{*}{ Variables } & \multirow[b]{2}{*}{ Overall $(n=152)^{\mathrm{a}, \mathrm{b}}$} & \multicolumn{2}{|c|}{ Stratified by Prior Treatment } & \multicolumn{2}{|c|}{ Stratified by LOT } \\
\hline & & $\overline{\text { Post-IO }(n=65)^{\mathrm{a}, \mathrm{b}}}$ & Post-TKI $(n=75)^{\mathrm{a}, \mathrm{b}}$ & LOT $2 / 3^{a}$ & LOT $4+^{\mathrm{a},}$ \\
\hline $\mathrm{N}$ & 152 & 65 & 75 & 67 & 85 \\
\hline Events (\%) & $77(50.7)$ & $36(55.4)$ & $36(48.0)$ & $36(53.7)$ & $41(48.2)$ \\
\hline Median $(95 \% \mathrm{CI})$ in months & $13.9(9.5,16.5)$ & $13.9(8.4,21.3)$ & $14.8(7.8,16.5)$ & $12.1(8.4,17.0)$ & $14.8(8.9,22.5)$ \\
\hline Min, Max & $0.2,43.8$ & $0.6,43.8$ & $0.2,34.5$ & $0.2,43.8$ & $0.2,34.6$ \\
\hline \multicolumn{6}{|c|}{ Overall survival probability, $\%(95 \% \mathrm{CI})$} \\
\hline 6 months & $70.8(62.5,77.6)$ & $72.5(59.5,81.9)$ & $67.7(55.1,77.4)$ & $72.8(59.8,82.1)$ & $69.2(57.6,78.2)$ \\
\hline 12 months & $52.7(43.4,61.1)$ & $52.7(38.7,65.0)$ & $50.4(37.0,62.4)$ & $50.2(36.0,62.8)$ & $54.7(42.2,65.5)$ \\
\hline 18 months & $37.5(27.9,47.0)$ & $37.7(24.0,51.3)$ & $35.1(21.3,49.3)$ & $34.8(21.4,48.6)$ & $39.3(26.0,52.4)$ \\
\hline 24 months & $28.5(18.3,39.5)$ & $25.7(12.5,41.1)$ & $35.1(21.3,49.3)$ & $27.9(15.0,42.3)$ & $27.5(12.5,44.9)$ \\
\hline
\end{tabular}

Abbreviations: CI, confidence interval; IO, immuno-oncology therapy; LADMF, Limited Access Death Master File; LOT, line of therapy;

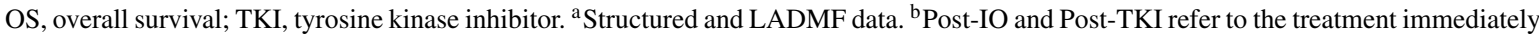
prior to Len/Eve.

treatment patterns and clinical outcomes of Len/Eve for a heavily pre-treated advanced/metastatic RCC in a community oncology setting.

Our study of 152 patients with advanced/metastatic RCC treated with $2 \mathrm{~L}+$ Len/Eve had similar demographic and clinical characteristics as reported in previous clinical trials and retrospective studies [7, 15-18]. Our study included a majority of male patients $(73.7 \%)$ who identified as Caucasian (78.9\%) with a median age of 63.4 years. These patients were heavily treated prior to Len/Eve with IO-based treatments (45.4\%), which were not available during the 
time of the clinical trials $[6,13,15]$. A recent retrospective study by Wiele et al. (2019) identified patients with advanced/metastatic RCC treated with Len $(n=10)$ or Len/Eve $(n=30)$ who had received at least two prior lines of therapy [14]. These patients had a median follow-up of 11.6 months $(95 \% \mathrm{CI}$ : 10.6, NA), which was similar to our median followup from index date of 7.5 months (range 0.2-43.8). Both retrospective studies reported a shorter median follow-up duration than patients participating in clinical trials $[7,15,16]$.

The median OS we report in our study [13.9 months (95\% CI: 9.5-16.5)] and the 12-month survival probability of $52.7 \%$ (95\% CI: 43.4-61.1) were comparable to those by Wiele et al. (2019), who reported a median OS of 10.8 months $(95 \% \mathrm{CI}$ : 5.4-13.3) and a 12-month survival probability of 46.0\% (95\% CI, 32.0-66.0) [14]. Our study also adds valuable OS data for post-IO and post-TKI therapy subgroups. OS times for patients in the post-IO and post-TKI subgroups were 13.9 months (95\% CI: 8.4-21.3) and 14.8 (95\% CI: 7.8-16.5) months, respectively.

This study population included a small proportion of patients with non-clear cell RCC (9.2\%), but stratified analysis was not assessed on this subset of patients due to the small sample size. However, this population tends to be treated similarly to clear cell RCC and historical data have shown that targeted therapies are the most commonly used agents for this population $[19,20]$. In a recent phase 2 clinical trial, Hutson et al. (2021) assessed the safety and efficacy of Len/Eve in patients with advanced non-clear cell RCC and found that Len/Eve had a favorable clinical profile, with a median OS of 15.6 months $(95 \% \mathrm{CI}$ : 9.2-NE) [21]. This suggests that our results can prove meaningful for patients with non-clear cell RCC.

To our knowledge, our study is the largest retrospective study to examine treatment patterns and outcomes of patients with advanced/metastatic RCC who received Len/Eve in the $\geq 2 \mathrm{~L}$ setting using realworld data, and provides important survival outcomes in heavily pre-treated populations such as the population in our study. Despite differences seen between our population and those of published clinical trials $[7,15,16]$, our results suggest favorable outcomes for patients who have been previously treated with IO and TKI therapies. Additional studies are warranted to increase the body of real-world data for patients with advanced/metastatic RCC, particularly to assess differences in outcomes for those who received prior TKI-based therapies versus prior IO-based therapies.

\section{Limitations}

An important limitation of the study is that not all patients in the study population had chart review data available. Data on ORR, PFS, and treatment related adverse events, which are found in chart review but not in structured EHR fields, were therefore not available for the entire study population. Also, while the median OS of nearly 14 months is encouraging for a heavily pretreated population, other factors besides Len/Eve treatment may be involved. Patients treated with $2 \mathrm{~L}+$ may have a more indolent tumor biology compared with patients who have progressive disease and received only one line of therapy. A prospective study with an appropriate control treatment arm would be a more relevant approach to determine the relationship of Len/Eve with improved survival.

Several other limitations must be noted. First, the study data originated within The US Oncology Network and Onmark clinics; therefore, the results may not necessarily be generalizable to all patients receiving Len/Eve for advanced RCC in the United States. Second, documentation bias could have resulted from data omissions or errors that can occur in retrospective observational studies. In addition, some structured data were not documented for several key variables (sarcomatoid features, history of nephrectomy, IMDC score). Finally, due to limitations of the data, dose reductions and toxicity were not captured. Future research should aim to assess these variables in a real-world setting. Still, despite these limitations, our study is one of the largest in a real-world setting, providing further information on clinical outcomes associated with Len/Eve treatment.

\section{CONCLUSION}

The results of this retrospective study demonstrated the clinical effectiveness of Len/Eve in an advanced/metastatic RCC population in US community oncology practices in both a post-IO and post-TKI setting. Future studies can supplement these findings by investigating the patient characteristics associated with Len/Eve outcomes in clinical practice. At the same time, further studies are needed to rule out other factors possibly associated with survival outcomes in this patient population.

\section{ACKNOWLEDGMENTS}

The authors would like to thank the patients and all investigational site members involved in this study, specifically Janet Espirito, PharmD of Ontada and 
Shrividya Iyer, $\mathrm{PhD}$ of Eisai Inc. for their help guiding the study design, as well as Lisa Kaspin-Powell, $\mathrm{PhD}$ of Ontada for modifying the manuscript.

\section{FUNDING}

This study was funded by Eisai Inc., Woodcliff Lake, NJ, USA, and Merck Sharp \& Dohme Corp., a subsidiary of Merck \& Co., Inc., Kenilworth, NJ, USA. The sponsors of this study were involved in the study design, interpretation of data, in the writing of the report, and in the decision to submit the article for publication. Medical writing support for the manuscript was provided by Ontada, Woodlands, TX, USA.

\section{AUTHOR CONTRIBUTIONS}

Concept and design: N. Vogelzang, Y. Wang, Y. Wan, Y. Xie, N. Robert, and N. Tannir. Collection and assembly of data: A. Monnette, Y. Wang, and Y. Xie. Data analysis and interpretation: All authors. Manuscript writing: All authors. Final approval of manuscript: All authors.

\section{CONFLICT OF INTEREST DISCLOSURE}

The authors declare the following financial interests/personal relationships which may be considered as potential competing interests: Nicholas J. Vogelzang reports grants and personal fees from Ontada and Eisai Inc., during the conduct of the study. Outside the submitted work but during the 36 months prior to publication, Dr. Vogelzang reports grants and personal fees from Amgen, Aravive, Astra Zeneca, Bristol-Myers Squibb, Calithera Bioscience, Caris, Exelixis, Inc, Nektar Therapeutics, and Novartis. Alisha Monnette reports that she has conducted contract work with Eisai Inc, during the conduct of the study and has also conducted contract work with Ipsen and Bristol-Myers Squibb outside the submitted work but during the 36 months prior to publication. Yunfei Wang reports that he has conducted contract work with Eisai, Inc, during the conduct of the study and has also conducted contract work with Ipsen and Bristol-Myers Squibb outside the submitted work but during the 36 months prior to publication. Yin Wan is an employee of Eisai Inc. Nicholas J. Robert reports grants from Eisai during the conduct of the study. Nizar Tannir reports personal fees from Eisai Inc., during the conduct of the study. Outside the submitted work but during the 36 months prior to publication, Dr. Tannir reports grants and personal fees from Bristol-Myers Squibb, Pfizer, Nektar Therapeutics, Exelisis, Inc, and Calithera Bioscience, along with personal fees from Eli Lilly, Oncorena, Surface Oncology, Novartis, and Ipsen. N. Vogelzang and N. Tannir are Editorial Board members of this journal, but were not involved in the peer-review process of this manuscript nor had access to any information regarding its peer-review.

\section{ETHICS APPROVAL}

Institutional Review Board and Compliance/Privacy approval was gained prior to initiation of the retrospective research. Since this project involved the analysis of existing data and records, study information was analyzed in such a manner that research participants could not be directly identified. Patient informed consent was not required due to the nature of the study design. Thus, exemption status and a waiver of informed consent were approved by The US Oncology, Inc. Institutional Review. Data were handled in compliance with HIPAA and Health Information Technology for Economic and Clinical Health (HITECH).

\section{REFERENCES}

[1] Siegel RL, Miller KD, Fuchs HE, Jemal A. Cancer statistics, 2021. CA Cancer J Clin. 2021;71(1):7-33.

[2] National Cancer Institute Surveillance, Epidemiology, and End Results Program. Cancer Stat Facts: Kidney and Renal Pelvis Cancer. 2021 [updated 2021. Available from: https://seer.cancer.gov/statfacts/html/kidrp.html.

[3] Tannir NM, Pal SK, Atkins MB. Second-line treatment landscape for renal cell carcinoma: a comprehensive review. The Oncologist. 2018;23(5):540-55.

[4] Kahl K. NCCN Recommends Frontline Lenvatinib/Pembrolizumab for Advanced RCC: Cancer Network; 2021 [Available from: https://www.cancernetwork.com/ view/nccn-recommends-frontline-lenvatinib-pembrolizum ab-for-advanced-rcc.

[5] FDA. FDA approves lenvatinib plus pembrolizumab for advanced renal cell carcinoma: U.S. Food \& Drug Administration; 2021 [Available from: https://www.fda.gov/drugs/ resources-information-approved-drugs/fda-approves-lenva tinib-plus-pembrolizumab-advanced-renal-cell-carcinoma.

[6] Drugs.com. FDA approves Lenvima (lenvatinib) for the treatment of patients with advanced renal cell carcinoma 2016 [Available from: https://www.drugs.com/newdrugs/ fda-approves-lenvima-lenvatinib-patients-advanced-renalcell-carcinoma-4382.html.

[7] Motzer RJ, Hutson TE, Glen H, Michaelson MD, Molina A, Eisen T, et al. Lenvatinib, everolimus, and the combination in patients with metastatic renal cell carcinoma: a 
randomised, phase 2, open-label, multicentre trial. Lancet Oncol. 2015;16(15):1473-82.

[8] Martinez Chanza N, Xie W, Asim Bilen M, Dzimitrowicz H, Burkart J, Geynisman DM, et al. Cabozantinib in advanced non-clear-cell renal cell carcinoma: a multicentre, retrospective, cohort study. Lancet Oncol. 2019;20(4):581-90.

[9] Peverelli G, Raimondi A, Ratta R, Verzoni E, Bregni M, Cortesi E, et al. Cabozantinib in renal cell carcinoma with brain metastases: safety and efficacy in a real-world population. Clin Genitourin Cancer. 2019;17(4):291-8.

[10] Stukalin I, Wells JC, Graham J, Yuasa T, Beuselinck B, Kollmansberger $\mathrm{C}$, et al. Real-world outcomes of nivolumab and cabozantinib in metastatic renal cell carcinoma: results from the International Metastatic Renal Cell Carcinoma Database Consortium. Curr Oncol. 2019;26(2):e175-e9.

[11] Campbell MT, Bilen MA, Shah AY, Lemke E, Jonasch E, Venkatesan AM, et al. Cabozantinib for the treatment of patients with metastatic non-clear cell renal cell carcinoma: A retrospective analysis. European journal of cancer (Oxford, England : 1990). 2018;104:188-94.

[12] Gross-Goupil M, Fléchon A, Chevreau C, Topart D, Gravis $\mathrm{G}$, Oudard S, et al. Real-world data of cabozantinib in patients with VEGF-refractory metastatic renal cell carcinoma (mRCC): results from the French Early Access Program (CABOREAL) American Society of Clinical Oncology Genitourinary Cancers Symposium; February 1315, 2020; San Francisco, CA, USA.

[13] Hamieh L, Beck RL, Le VH, Hsieh JJ. The efficacy of lenvatinib plus everolimus in patients with metastatic renal cell carcinoma exhibiting primary resistance to front-line targeted therapy or immunotherapy. Clin Genitourin Cancer. 2020;18(4):252-7 e2.

[14] Wiele A, Ross J, Chahoud J, Lim Z, Xiao L, Campbell MT, Shah AY, Saavedra A, Jonasch E, Tannir N. Lenvatinib (Len) alone or in combination with everolimus (Eve) in heavily pretreated patients (pts) with metastatic renal cell carcinoma (mRCC) after immune checkpoint inhibitors (ICI) and VEGFR-targeted therapies: A single-institution experience. Annals of Oncology. 2019;30(5):v390.
[15] Matsubara N, Naito Y, Nakano K, Fujiwara Y, Ikezawa H, Yusa $\mathrm{W}$, et al. Lenvatinib in combination with everolimus in patients with advanced or metastatic renal cell carcinoma: A phase 1 study. Int J Urol. 2018;25(11):922-8.

[16] Molina AM, Hutson TE, Larkin J, Gold AM, Wood K, Carter D, et al. A phase $1 \mathrm{~b}$ clinical trial of the multitargeted tyrosine kinase inhibitor lenvatinib (E7080) in combination with everolimus for treatment of metastatic renal cell carcinoma (RCC). Cancer Chemother Pharmacol. 2014;73(1):181-9.

[17] Shah AY, Kotecha RR, Lemke EA, Chandramohan A, Chaim JL, Msaouel P, et al. Outcomes of patients with metastatic clear-cell renal cell carcinoma treated with second-line VEGFR-TKI after first-line immune checkpoint inhibitors. European journal of cancer (Oxford, England: 1990). 2019;114:67-75.

[18] Glen H, Puente J, Heng DYC, Rha SY, Li D, Stepan DE, et al. A phase 2 trial of lenvatinib $18 \mathrm{mg}$ versus $14 \mathrm{mg}$ once daily (QD) in combination with everolimus (5mg QD) in renal cell carcinoma (RCC) after 1 prior VEGF-targeted treatment. Journal of Clinical Oncology. 2018;36(6_suppl):TPS707TPS.

[19] Ahrens M, Scheich S, Hartmann A, Bergmann L, IAG$\mathrm{N}$ Interdisciplinary Working Group Kidney Cancer of the German Cancer Society. Non-Clear Cell Renal Cell Carcinoma - Pathology and Treatment Options. Oncol Res Treat. 2019;42(3):128-35.

[20] Tannir NM, Jonasch E, Albiges L, Altinmakas E, Ng CS, Matin SF, et al. Everolimus Versus Sunitinib Prospective Evaluation in Metastatic Non-Clear Cell Renal Cell Carcinoma (ESPN): A Randomized Multicenter Phase 2 Trial. Eur Urol. 2016;69(5):866-74.

[21] Hutson TE, Michaelson MD, Kuzel TM, Agarwal N, Molina AM, Hsieh JJ, et al. A Single-arm, Multicenter, Phase 2 Study of Lenvatinib Plus Everolimus in Patients with Advanced Non-Clear Cell Renal Cell Carcinoma. Eur Urol. 2021;80(2):162-70. 